\title{
Examining neural correlates of skill acquisition in a complex videogame training program
}

\author{
Ruchika S. Prakash ${ }^{1}$, Angeline A. De Leon ${ }^{1}$, Lyla Mourany ${ }^{1}$, Hyunkyu Lee ${ }^{2}$, Michelle W. Voss ${ }^{2}$, \\ Walter R. Boot ${ }^{3}$, Chandramallika Basak ${ }^{4}$, Monica Fabiani ${ }^{2}$, Gabriele Gratton ${ }^{2}$ and Arthur F Kramer ${ }^{2}$ \\ 1 Department of Psychology, The Ohio State University, Columbus, OH, USA \\ 2 Department of Psychology and Beckman Institute for Advanced Science and Technology, University of Illinois, Champaign-Urbana, IL, USA \\ ${ }^{3}$ Department of Psychology, Florida State University, Tallahassee, FL, USA \\ ${ }^{4}$ Department of Brain and Behavioral Sciences, University of Texas at Dallas, Dallas, TX, USA
}

\section{Edited by:}

Torsten Schubert, Ludwig Maximilians University Munich, Germany

\section{Reviewed by:}

Claude Alain, Rotman Research

Institute, Canada

Leighton B. Hinkley, University of

California at San Francisco, USA

${ }^{*}$ Correspondence:

Ruchika S. Prakash, Department of Psychology, The Ohio State University, 1835 Neil Avenue, Columbus, $\mathrm{OH}$ 43210, USA. e-mail:

prakash.30@osu.edu
Acquisition of complex skills is a universal feature of human behavior that has been conceptualized as a process that starts with intense resource dependency, requires effortful cognitive control, and ends in relative automaticity on the multi-faceted task. The present study examined the effects of different theoretically based training strategies on cortical recruitment during acquisition of complex video game skills. Seventy-five participants were recruited and assigned to one of three training groups: (1) Fixed Emphasis Training (FET), in which participants practiced the game, (2) Hybrid Variable-Priority Training (HVT), in which participants practiced using a combination of part-task training and variable priority training, or (3) a Control group that received limited game play. After $30 \mathrm{~h}$ of training, game data indicated a significant advantage for the two training groups relative to the control group. The HVT group demonstrated enhanced benefits of training, as indexed by an improvement in overall game score and a reduction in cortical recruitment post-training. Specifically, while both groups demonstrated a significant reduction of activation in attentional control areas, namely the right middle frontal gyrus, right superior frontal gyrus, and the ventral medial prefrontal cortex, participants in the control group continued to engage these areas posttraining, suggesting a sustained reliance on attentional regions during challenging task demands. The HVT group showed a further reduction in neural resources post-training compared to the FET group in these cognitive control regions, along with reduced activation in the motor and sensory cortices and the posteromedial cortex. Findings suggest that training, specifically one that emphasizes cognitive flexibility can reduce the attentional demands of a complex cognitive task, along with reduced reliance on the motor network.

Keywords: skill acquisition, training strategies, attentional control, functional MRI

\section{INTRODUCTION}

The ability of humans to acquire both simple and complex skills is a universal feature of human behavior, one that starts early in life (Piaget, 1954) and enables the development of a repertoire of cognitive, motor, and perceptual processes essential for successful human functioning. The study of skill acquisition has been the focus of research for many decades now, with many theorists proposing that skill acquisition involves an ordered series of stages, with earlier stages focused on effortful, controlled processing, characterized by greater cognitive and executive control, and later stages resulting in automaticity of behavior, depending on fewer resources and little effort (Fitts and Posner, 1967; Schneider and Shiffrin, 1977; Ackerman, 1988). An important variable in the learning of complex skills is the differential influence of training strategies on learning rate, with more efficient training regimes characterized both by a faster acquisition of the skill involved, and by a resourceful utilization of the various skill dimensions, resulting in efficient performance. The Learning Strategies Initiative (Donchin et al., 1989) outlined a series of training strategies that were examined for their ability to enhance complex skill acquisition, as implemented in a multi-faceted videogame, Space Fortress (SF). Training strategies included repeated practice on the entire task (Fixed Emphasis Training, FET), which has been the predominant mode of training and cognitive rehabilitation across various clinical populations (e.g., Chiaravalloti et al., 2005; Erickson et al., 2007), part-task training, involving principled decomposition of the complex videogame into skill and knowledge components and training individuals on the sub-parts rather than on the integrated game (Frederiksen and White, 1989), and whole-task training with variable priority (Variable Priority Training, VPT), involving training on the integrated complex task, with changing emphasis on the sub-components of the game throughout training (Gopher et al., 1989; see Fabiani et al., 1989, for a comparison of these training regimes).

The recent resurgence of interest in cognitive training to enhance cognitive vitality and neural plasticity (Boot et al., 2011; Slagter et al., 2011) has led to a re-examination of the prophylaxis offered by various training strategies for the faster acquisition of 
complex skills (Basak et al., 2008; Boot et al., 2010; Lee et al., 2012), cortical reorganization as evidenced by altered brain activity and connectivity (Kantak et al., 2010; Maclin et al., 2011; Voss et al., 2011), and transfer to novel tasks (Boot et al., 2010; Stern et al., 2011; Lee et al., 2012). Prioritizing different aspects of a complex task, while performing the integrated task (VPT), has been found to be beneficial for dual-task performance (Kramer et al., 1995, 1999; Bherer et al., 2008), faster learning and higher level of mastery on the videogame SF (Fabiani et al., 1989; Boot et al., 2010), and better working memory performance in older adults (Stern et al., 2011).

Adding to the behavioral literature have been recent investigations of the neural correlates of variable priority versus FET (Kantak etal., 2010; Voss etal., 2011). Employing repetitive transcranial magnetic stimulation (rTMS), Kantak et al. (2010) provided evidence for the dependence of the two practice regimes on separable cortical areas for motor memory consolidation. While application of rTMS on the dorsolateral prefrontal cortex post-variable practice resulted in attenuation of motor skill retention, it was interference with the primary motor cortices following constant practice that attenuated motor retention, thus providing evidence for the use of different cortical regions in consolidation based on the strategy implemented. Similarly, Voss et al. (2011) suggested the differential interaction of the declarative and procedural learning systems with higher-order attentional networks as a function of training strategies. After $20 \mathrm{~h}$ of training, the basal ganglia, associated with the learning system related to FET, and the medial temporal lobes (MTL) associated with the learning system related to VPT, both showed enhanced interaction with the fronto-parietal system. The interaction between the MTL and the fronto-parietal system in the VPT group is implicated in the increased capacity of working memory and attention (Craik et al., 1996; Olesen et al., 2004). Therefore, VPT trainees may be more efficiently utilizing their attentional network, suggesting that this training strategy involves more flexible attentional control. In addition, unique to FET, Voss et al. (2011) observed enhanced interactions between the MTL and the fronto-executive system. Given the increased interaction of the basal ganglia with the fronto-parietal system and the MTL with the fronto-executive system in the FET group, it appears that FET participants were concurrently utilizing two different cognitive control systems. The authors postulated this enhanced functional connectivity in the two attention systems to be indicative of a higher cognitive load for FET, which in turn, leads to a reliance on basal ganglia and procedural motor sequences to accomplish game performance. This unique pattern of functional connectivity in the attentional network of the FET group was thus indicative of increased engagement of attentional resources during game-play, which, relative to VPT, suggested an inefficient modulation of neural activity in attentional areas.

Research studies investigating the neurophysiological indices of skill acquisition as a function of training strategy also provide evidence for a greater increase in alpha frequency in the parttask training groups relative to the whole-task training group, providing evidence of attenuation of cognitive effort and attentional demands with an efficient training strategy (Smith et al., 1999). Recently, Maclin et al. (2011) also reported a decrease in P3 amplitude following training on the SF game for some components of the game. The investigators interpreted these results as providing evidence of greater allocation of attention to a secondary task post-training. Thus, evidence from behavioral, and neuroimaging studies provide consistent data on the superiority of training regimes that focus either on training different components of the task independently, or training that prioritizes selective aspects of a complex task within the context of the whole task during skill acquisition. In the present study, capitalizing on the benefits of part-task training and emphasis change training approaches, we examined the efficacy of a Hybrid Variable-Priority Training (HVT) approach to produce greater skill mastery (Gopher et al., 1994; Lee et al., 2012).

By combining both part-task training, which enables the breakdown of a complex task into small sub-component tasks which can then be individually mastered, and VPT, which enables participants to explore and learn new strategies and transfer subcomponents skills learned during part-task training to the integrated whole task, HVT exploits the benefits of both approaches, thus resulting in superior behavioral performance as compared to variable priority alone (Gopher et al., 1994). Seventy-five participants were recruited for the current study and randomized to one of three groups: (1) FET, in which participants practiced the game, (2) HVT, in which participants practiced using a combination of part-task training and VPT, or (3) a Control group that received limited game play. All participants played the videogame inside an MRI scanner pre- and post-training, and neural recruitment during game performance was compared across groups as a function of training.

We hypothesized that both training groups would achieve a greater level of skill mastery than the control group, as demonstrated by a greater behavioral improvement in game performance, along with a reduction in the recruitment of the lateral prefrontal regions known to subserve cognitive control operations (Miller and Cohen, 2001). We reasoned that repeated practice on a task for $30 \mathrm{~h}$ would involve a transition from the effortful, resourceintensive earlier stages of skill acquisition to a stage of relative automaticity, characterized by a reduction in the need to exert top-down control, along with a concomitant decrease in the activity of the prefrontal cortices (Poldrack et al., 2005). In order to examine the effects of practice on the videogame on behavioral performance and neural recruitment, we merged data from the two training groups to evaluate first the effect of practice on the SF game, relative to a limited-contact control group. In our second set of analyses, we compared the two training groups directly to investigate the differential effect of strategy on behavior and cortical recruitment. A comparison of the two training strategies, we hypothesized, would show significantly greater skill mastery for the HVT group relative to the FET group, along with continued recruitment of the regions of the prefrontal cortices in the FET group, relative to the HVT group, demonstrating a greater need to exert top-down control in the face of sub-optimal strategies acquired due to simple practice on the complex task. In addition, we hypothesized that game play in FET participants would depend upon the motor network, involving the primary motor cortices and the supplementary motor areas, reflecting learning based on routine behavior and fixed skills (Myers et al., 2003). 


\section{MATERIALS AND METHODS PARTICIPANTS}

Seventy-five young adults were recruited for the current study from the Champaign-Urbana community via flyers and announcements posted throughout the University of Illinois campus. Interested participants were asked to fill out a survey collecting basic demographics, and measuring videogame play during the last 12 months (available at http://spacefortress.blogspot.com). Participants were excluded from the study if they indicated videogame play of more than $4 \mathrm{~h}$ per week, presence of any psychiatric or neurological condition, and left-handedness as assessed by the Edinburgh Handedness Inventory. Participants meeting eligibility criteria were initially randomly divided across three groups: (1) FET, (2) HVT, or (3) a no-contact control group. Halfway through the recruitment process, the basic demographics of the three groups were checked to ensure that no systematic differences existed across groups in age or gender. All participants were paid $\$ 15$ an hour for their participation. The University of Illinois Institutional Review Board approved the study and all participants gave informed consent. Participant demographics for each of the three groups are displayed in Table 1 . The groups did not differ on any of the demographic variables.

Of these 75 participants, 72 completed the MRI session preand post-training. Two participants were excluded because of

Table 1 | Descriptive characteristics of participants in the three training groups (FET, HVT, and control) based on all 75 participants and on the sample of 66 participants used for the analyses reported in this paper.

$\begin{array}{lll}\text { Fixed Emphasis } & \text { Hybrid } & \text { Control group } \\ \text { Training (FET) } & \text { Variable-priority } & \\ & \text { Training (HVT) } & \end{array}$

\begin{tabular}{llll}
\hline $\mathbf{7 5}$ Participants & & & \\
$N$ & 25 & 25 & 25 \\
Age & $21.91(2.78)$ & $20.88(2.07)$ & $21.44(2.52)$ \\
Proportion male & 0.36 & 0.40 & 0.44 \\
Self-rated health & 5 & 5 & 5 \\
Year of education & $15.52(2.20)$ & $14.68(1.85)$ & $15.28(2.25)$ \\
Baseline score & -844.45 & -1034.78 & -988.39 \\
& $(2086.82)$ & $(1907.15)$ & $(1916.30)$ \\
66 Participants & & & \\
N & 23 & 22 & 21 \\
Age & $22(2.90)$ & $20.86(2.19)$ & $21.48(2.71)$ \\
Proportion male & 0.34 & 0.41 & 0.47 \\
Self-rated health & 5 & 5 & 5 \\
Year of education & $15.61(2.27)$ & $14.68(1.97)$ & $15.24(2.37)$ \\
Baseline score & -857.51 & -1102.02 & -860.40 \\
& $(1925.31)$ & $(1909.59)$ & $(1926.95)$
\end{tabular}

Standard deviations are within parentheses. No significant differences were found between the full sample and the subset of 66 participants on demographics or behavioral performance at Time 1 and Time 2. Total game score improvement was also not significantly different between the two groups. For self-related health, the scale was ranging from 1 for poor to 5 for excellent. problems in data acquisition. Four out of the remaining 70 participants were excluded from the current analyses because of excessive motion (greater than one functional voxel) in more than 10 functional $\mathrm{T}^{\star}$ images in all three runs of the fMRI data. All analyses were conducted with the remaining 66 participants, whose demographics are also presented in Table 1 . There were no statistically significant differences in age, gender, or education between the full sample and the subset that was analyzed for the current study. Please note that the behavioral data presented here have been previously reported in Lee et al. (2012), and that the current study focuses exclusively on the functional MRI data.

\section{STUDY PROCEDURES}

The present study employed a randomized controlled trial to examine the effects of training and training strategies on behavioral and neural functioning. All recruited participants were oriented to the game via a 20 -min instructional video that detailed the requirements of the game (video also available at http:// spacefortress.blogspot.com), followed by another 5-min summary video that reviewed the important rules. Following the video demonstration, all participants completed a pop-up quiz inquiring about instructions, and after ensuring that they had successfully understood the rules of the games and the operations involved, participants played six 3-min games. Following the game orientation session, all participants underwent a detailed cognitive assessment session (the results of which are reported in Lee et al., 2012), an event-related brain potential (ERP) session (which is not the focus of this manuscript), and a functional MRI session.

Participants successfully undergoing the assessment sessions were divided into three groups, two of which were training groups (FET and HVT) where participants completed fifteen 2-h sessions, resulting in $30 \mathrm{~h}$ of training on the videogame, SF. The third, control group received contact with the game at pre-training, after the training groups completed $10 \mathrm{~h}$ of training, and then again at post-training. Below we describe in brief the SF videogame, which was used as a platform in the current study to implement the different strategies and examine changes in cortical recruitment.

\section{Space Fortress}

The SF videogame was originally developed in a cognitive psychophysiology laboratory (Mané and Donchin, 1989) to provide a platform for the study of complex skill acquisition in an environment that was visually engaging, and modeled the complexities and multi-dimensionality of real-life tasks. As such, the SF game taps into perceptual, motor, executive and attentional skills, and thus lends itself as an ideal stage for the training of these various cognitive abilities, either through repeated practice on the whole game or training on different components to master the varied cognitive operations involved. This particular videogame has been used extensively in research studies (see Fabiani et al., 1989; Gopher et al., 1989; Mané and Donchin, 1989; Rabbitt et al., 1989; Boot et al., 2010; Maclin et al., 2011; Voss et al., 2011; Lee et al., 2012), and thus here we briefly discuss the game and outline the main components. A depiction of the SF game screen is presented in Figure 1A. 


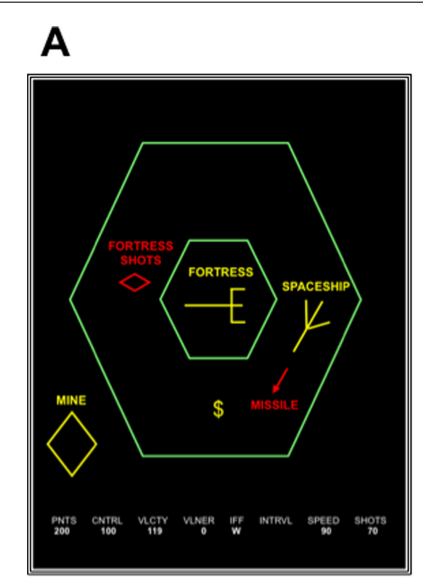

\section{B}

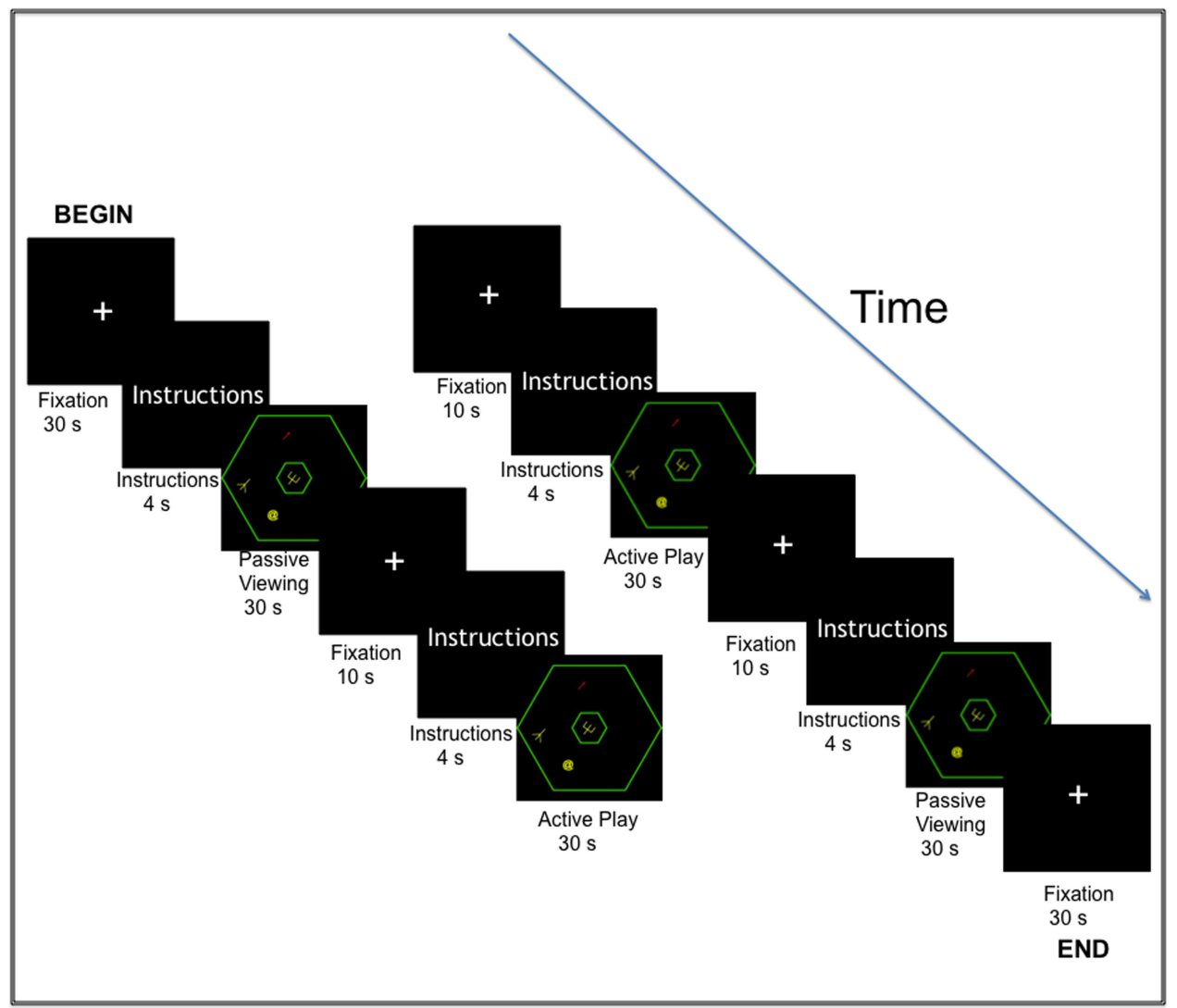

FIGURE 1 I Schematic representation of Space Fortress (A), along with a graphical description of the SF MRI task design with blocks of active play and passive viewing interspersed with fixation periods (B).

Players are awarded a total score for each game, which is the sum of four sub-scores (Control, Velocity, Speed, Points). The main task in the SF game is for the player, represented by a ship, to destroy a SF, which is located in the center of the screen within two hexagons, using missiles allotted to them. The ship flies in this environment with no brakes, so the player must exercise slight, precise movements of the joystick, keeping the ship in the large hexagon, and a failure to do so results in a reduction of Control scores. Successfully destroying the SF contributes to the Point subscore. Players are penalized if they improperly execute the series of missile launches required to destroy the fortress and if the fortress' missiles hit the ship.

In addition, throughout the game, participants also have to deal with mines and acquire bonus points and missiles. Diamondshaped foe or friend mines appear on the screen, with a corresponding letter that is displayed on the bottom of the screen indicating the friend or foe status of the mine. Correctly recognizing the mines and taking appropriate action contributes toward the Speed sub-score. However, if a mine is misidentified, the damage that the mine endures transfers to the ship and the player receives a deduction in the Speed sub-score.

Participants are also given opportunity to earn bonus missiles or bonus points, by constantly monitoring the appearance of a dollar sign in their field of play. To earn the bonus, participants are asked to stay vigilant of the appearance of a pair of dollar signs, and clicking the mouse buttons at the second, not the first dollar sign earns them bonus missiles or bonus points.

\section{Training groups}

The training groups employed in this study were modeled after the groups used in the Gopher et al.'s (1994) study to capitalize on the benefits of both part-task training and variable priority whole-task training to achieve accelerated skill acquisition. Our first training group, the FET group received no formal strategy training and were simply instructed to concentrate on obtaining as high a total score as possible, while focusing on the different components of SF equally. All participants in this group played thirty 3-min games of SF each session for 15 sessions.

The second training group, referred to as the HVT group combined both part-task training and VPT. A combination of part-task and VPT (combined sessions) was employed in the first five sessions, while exclusive VPT was used in the last 10 sessions. During the first hour and $10 \mathrm{~min}$ of the combined sessions, part-task training was employed, in which players practiced a specific component of SF that was presented separately from the rest of the game. For example, in a given game during part-task training, 
participants might be presented with the task of just navigating the ship or just aiming and firing. During the remaining $50 \mathrm{~min}$ of the combined session, participants in the HVT group were instructed to employ VPT. Participants played SF in its entirety with the goal of focusing on the specific skill that was previously learned in the part-task training and scoring as high a total score as possible on that particular component. The details of the part-task training are described in Table 2 . In the last 10 sessions employing the VP strategy, participants were asked to emphasize different components of the game sequentially. In these sessions, participants completed five practice blocks of six trials emphasizing the four sub-scores.

Both training groups, at the start and end of every session, played three test games where total score was emphasized, and these data were used as behavioral data for pre- and post-game scores and used in all behavioral analyses and brain-behavior correlations.

\section{BEHAVIORAL ANALYSES}

To analyze the effects of videogame training on improvement on SF game performance, quantified by total score across all four components, we conducted two repeated-measures ANOVAs with

Table 2 | Details of the part tasks implemented in the first five sessions.

\section{Part-training details}

1. Destroy Fortress by shooting

2. Slow down a ship

3. Aiming

4. Aiming and firing

5. Navigating a ship in trajectory 1

6. Navigating a ship in trajectory 2

7. Navigating a ship in trajectory 3

8. Navigating a ship in big hexagon

9. Navigating a ship in small hexagon

10. Navigating a ship in hexagon and aiming

11. Navigating a ship in hexagon, aiming, and firing

12. Navigating a ship in hexagon, aiming, and firing on the shooting fortress

13. Ship control only

14. Full game without bonus and mine

15. Mine control only

16. Bonus control only

17. Mine and bonus control

18. Mine and ship control

19. Bonus and ship control

20. Full game without bonus control

21. Full game without mine control

The first two part tasks were implemented only in the first two sessions, while the remaining part-tasks were implemented in all five part-task training sessions. time (pre-training, post-training) as the within-subjects factor and group as the between-subjects factor ${ }^{1}$. Gender was included as a covariate in the ANOVAs, as previous research had shown that gender differences exist in videogame performance (Terlecki and Newcombe, 2005; Feng et al., 2007). In the first ANOVA, to examine the influence of training on game performance, we merged the two training groups into one and tested whether training on the SF game was associated with improvements in total game score, relative to the control group. This ANOVA included time (pre-training, post-training) as a within-subjects factor and group (Control, Training) as a between-subjects factor.

In order to examine the influence of training strategy on game improvement, we conducted a second repeated-measures ANOVA with time (pre-training, post-training) as a within-subjects factor and training strategy as a between-subjects factor (HVT, FET). All behavioral data were analyzed using SPSS 17.0 for Mac.

\section{fMRI DATA ACQUISITION AND TASK PARAMETERS}

Participants were scanned in a 3-Tesla Siemens Allegra head-only scanner at the Beckman Institute for Advanced Science and Technology at University of Illinois. Structural T1-weighted images were acquired using a 3-D magnetization prepared rapid gradient echo imaging (MPRAGE) protocol with 144 contiguous axial slices, collected in ascending order, echo time $(\mathrm{TE})=3.87 \mathrm{~ms}$, repetition time $(\mathrm{TR})=1800 \mathrm{~ms}$, field of view $(\mathrm{FOV})=256 \mathrm{~mm}$, acquisition matrix $160 \mathrm{~mm} \times 192 \mathrm{~mm}$, slice thickness $=1.3 \mathrm{~mm}$, and flip angle $=8^{\circ}$.

Functional $\mathrm{T}^{*}$ weighted images were acquired using a fast echo-planar imaging (EPI) sequence with blood oxygenation level-dependent (BOLD) contrast $(64 \times 64$ matrix, $3.4 \mathrm{~mm} \times 3.4 \mathrm{~mm} \times 4.0 \mathrm{~mm}$ voxel size, $\mathrm{TR}=2000 \mathrm{~ms}$, $\mathrm{TE}=25 \mathrm{~ms}$, and flip angle $=80^{\circ}$, number of slices $=28$ ). Using a MRI-compatible joystick, all participants completed three full runs of the SF game during MRI scanning at pre- and postassessment. Presentation of SF during the MRI session was based on a block design consisting of two 30-s blocks of active gameplay and two 30-s blocks of passive viewing, interspersed with 10 -s fixation periods and 4-s of instructions. During blocks of active game-play, participants were instructed to play the game like they would play it in the laboratory, and during passive view, participants watched a video of an expert playing the videogame. A total of 115 volumes were collected for each functional run. A depiction of the SF MRI task design is presented in Figure 1B.

\footnotetext{
${ }^{1}$ Two separate ANOVAs were conducted to examine separately the effects of practice and training strategy on game score improvement. Given that the VP and FP groups both practiced the game for $30 \mathrm{~h}$, we examined if practice on the game would result in overall improvement in game performance and thus for the first ANOVA data from the two groups were merged to examine this hypothesis. However, to examine if unequal differences in sample size between the practice groups (FP and VP combined) and the control group could result in significant effects on the ANOVA, we also conducted a repeated-measures ANOVA with all three groups in the model (Control, HVT, FET), with time as the within-Ss factor and gender as the covariate to examine the effects on a time $\times$ group interaction. With the three groups as the between Ss factor, we find a main effect of time $[F(1,62)=21.19$, $p<0.001]$, group $[F(2,62)=6.54, p<0.005]$, and a significant time $\times$ group effect $[F(2,62)=23.03, p<0.001]$.
} 


\section{fMRI ANALYSES}

Neuroimaging data were analyzed using FSL 4.1 and FEAT (fMRI Expert Analysis Tool). Images were corrected for motion using a rigid-body algorithm in MCFLIRT, and smoothed with a Gaussian high-pass filter of $100 \mathrm{~s}$. Structural T1-weighted images were skull-stripped using a robust deformable brain extraction technique (BET). The skull-stripped images for each participant were transformed to a standard Montreal Neurological Institute (MNI) space and then spatially registered to each participant's highresolution scan. All participants, as mentioned above, played three full runs of the SF game. Given that participants were required to play the videogame with a MRI-compatible joystick inside the fMRI scanner, we noticed significant motion for many participants across different runs of the game. For each participant, we decided to exclude one run with the lowest signal-to-noise ratio (SNR) and motion greater than 1 functional voxel space $(3.475 \mathrm{~mm})$ in 10 or more volumes. Final analyses were conducted with two runs of the SF game for each participant at pre- and post-training.

Following pre-processing, the functional data collected during the presentation of the SF game were convolved with a doublegamma function to model the response for each condition (active game playing and passive viewing). This first-level analysis, done separately for each participant for the two functional runs, resulted in voxel-wise parameter estimate maps for the entire brain for each condition (active, passive), and for the direct comparison between the conditions (Active $>$ Passive). These parameter estimate maps and variance maps from the two functional runs were then aggregated within subject (across the two functional runs) for greater statistical power, using ordinary least squares (OLS) in FSL's FEAT tool. This was done separately for Time 1 and Time 2 to examine recruitment of cortical regions during active game play before and after the intervention for each individual participant.

Finally, the mean individual-level statistical maps from the two time-points were forwarded to a third-level fixed effects, individual-level longitudinal analysis to examine the influence of training on neural recruitment during active game playing and passive viewing separately for each individual participant. This was done using OLS in FSL's FEAT tool. This third-level analysis resulted in statistical maps representing activation during active game playing and passive viewing at pre-training, posttraining and the contrast between the two time-point for each individual participant. These parameter estimates were forwarded to two separate fourth-level, mixed-effects analyses, paralleling the behavioral analyses that considered between-subject variation. Both these analyses were conducted using FLAME (fMRIB's Local Analysis of Mixed Effects). All statistical maps were thresholded at a voxel-wise $z$-score of $2.33(p<0.01)$ and a cluster-wise threshold of $p<0.05$, with a minimum cluster size of five hundred and twenty-two $2 \mathrm{~mm}^{3}$ voxels.

The first higher-level analysis was conducted to locate regions of cortex that showed an influence of training on cortical recruitment during active game play. For this, we examined the contrast of Active $>$ Passive game play for the three groups. Here, we were primarily interested in changes in neural recruitment following post-training in the control group relative to the training groups. We examined changes at post-training relative to pre-training $(\mathrm{T} 2>\mathrm{T} 1)$ in the contrasts of Control $>$ Training and Training $>$ Control. Regions of interest (ROIs) from this whole-brain analysis comparing the control group to the training groups were identified to examine associations with behavioral improvement in the SF game. Specifically, statistical peaks in separable anatomical regions as demarcated by the HarvardOxford cortical atlas, packaged with the FSL software package (FSL 4.1.4, FMRIB's Software Library, www.fmrib.ox.ac.uk/fsl) in the contrast of Control $>$ Training from T2 $>$ T1 were taken to examine brain-behavior relationships. We then created a 14-mm sphere around each of these statistical peaks and extracted percent signal change for the contrast of Active $>$ Passive for both pre- and post-training, to examine associations with game improvement.

In addition, we were also interested in examining how cortical recruitment in these regions differed as a function of training strategy. We extracted percent signal change from these regions at pre- and post-training and conducted an independent samples $t$-test comparing differences in change in cortical recruitment from pre- to post-training between the two training groups.

The second higher-level analysis was conducted to directly compare cortical recruitment for the two training strategies to better understand the neural correlates involved with accelerated skill acquisition in the HVT group relative to the FET group. The above ROI analysis represented a focused examination of the changes in cortical activation in the two training groups in functional regions that showed continued activation in the control group, relative to the training groups. In this whole-brain analysis, independent of the control group, we examined differential cortical recruitment in the two training groups at post-training, relative to pre-training. Statistical peaks in this contrast were also taken to create ROIs for examining brain-behavior associations.

\section{RESULTS BEHAVIORAL RESULTS}

The effect of training on behavioral performance was examined using a repeated-measures ANOVA with time (pre-training, posttraining) as a within-subjects factor and group (control, training) as a between-subjects factor. We found a main effect of time $[F(1,63)=15.4, p<0.01]$, indicating that all groups had significant improvement in total game score from Time 1 to Time 2, along with a significant Time $\times$ Group interaction $[F(1,63)=40.0$, $p<0.01$, suggesting that training across both strategies was beneficial for behavioral performance in the SF game, relative to the control group (Figure 2).

To examine whether HVT as a training strategy was related to greater levels of game mastery in comparison to FET, we contrasted HVT and FET using a repeated-measures ANOVA, using the average total score from SF at Time 1 and Time 2 as a within-subjects factor and group as a between-subjects factor. We found a main effect of time $[F(1,42)=18.8, p<0.01]$ as well as a significant Group $\times$ Time interaction $[F(1,42)=4.72$, $p<0.05]$, which indicated a greater benefit on SF game performance for the HVT group relative to the FET group. This suggests that a training strategy combining part-training with variable priority is more beneficial than practice alone on the SF game (Figure 2). 


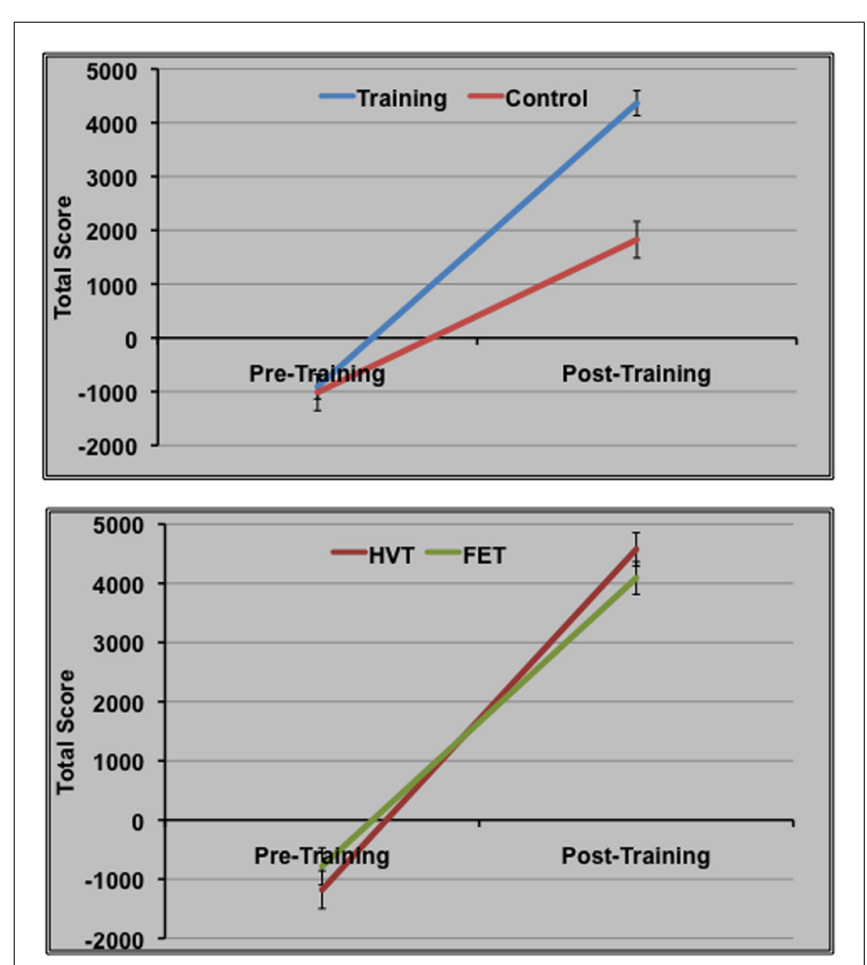

FIGURE 2 | Space Fortress behavioral data for the three groups at preand post-training.

\section{NEUROIMAGING RESULTS \\ Practice-related differences in cortical recruitment and associations with behavioral performance}

In order to examine the effects of practice on neural recruitment during active game play, we conducted a whole-brain analysis contrasting brain activation during the Active $>$ Passive condition at Time $2>$ Time 1, separately comparing the control group to the training groups. A contrast of the control group and the training groups (Control $>$ Training) showed decreased activation of the right middle frontal gyrus (rt. MFG), right superior frontal gyrus (rt. SFG), and ventral medial prefrontal cortex (vmPFC), for the training groups relative to the control group (Figure 3 ). Table 3 provides the $\max z$-stat values in MNI space for the peak voxels in this contrast. In line with our hypothesis, these results demonstrate that videogame training, in comparison to the control condition, results in a reduced need for activation of attentional areas during game-play (Figure 3). Statistical peaks in this contrast were taken to create ROIs, which were then examined for associations with behavioral performance. For this, we conducted partial correlations, controlling for the effects of gender between game improvement from pre- to post-training and percent signal change in regions identified in the contrast of Control $>$ Training. We found a negative relationship between game improvement and increase in activation in the right MFG $(r=-0.31, p<0.01)$ and a trend for a negative association for the right SFG $(r=-0.22$, $p=0.08$ ), such that individuals showing a greater increase in activation of these regions from pre- to post-training also demonstrated the lowest gains in game improvement.

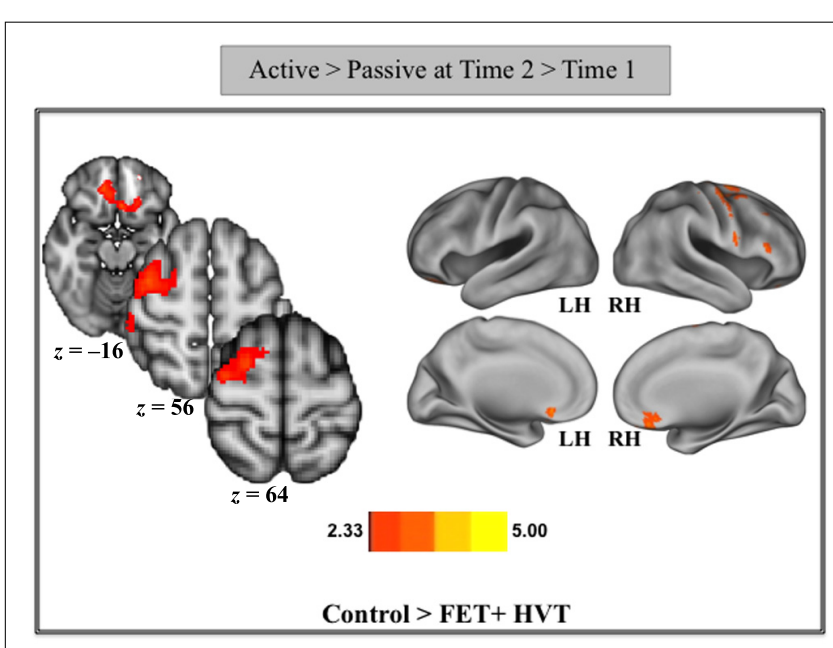

FIGURE 3 | Cortical areas recruited by the controls relative to the two training groups at post-training, when compared to pre-training. All axial slices are presented in radiological orientation.

Table 3 | Statistical peaks of cortical regions recruited during the Active $>$ Passive condition at Time $2>$ Time 1 contrasting the control group with the training groups (Control $>$ Training).

\begin{tabular}{|c|c|c|c|c|c|}
\hline \multirow[t]{2}{*}{ Anatomical region } & \multirow[t]{2}{*}{ Label } & \multirow{2}{*}{$\begin{array}{l}\text { Max } \\
\text { z-stat }\end{array}$} & \multicolumn{3}{|c|}{ MNI coordinates } \\
\hline & & & $X$ & $Y$ & $Z$ \\
\hline Right middle frontal gyrus & Rt. MFG & 3.39 & 44 & 0 & 56 \\
\hline Right superior frontal gyrus & Rt. SFG & 3.57 & 34 & 62 & 70 \\
\hline Ventral medial prefrontal cortex & vmPFC & 2.68 & 22 & -2 & 68 \\
\hline
\end{tabular}

\section{Training strategy-related differences in cortical recruitment and associations with behavioral performance}

The above identified functional ROIs from the contrast of Control $>$ Training were also examined for differences as a function of training strategy. As seen in Figure 4, the FET group showed greater increase in activation than the HVT group at Time 2 relative to Time 1 for all ROIs; however, significant increases in activation were noted for the right MFG and right SFG $(p<0.05)$ in comparison to HVT after training. This finding suggests that individuals in the FET group required continued activation of the prefrontal cortices in order to meet the demands of the SF game, whereas individuals in the HVT group showed reduced recruitment of these prefrontal regions as a function of the training strategy.

While the above discussed ROI analysis represented a focused examination of the effects of training strategy on the recruitment of cortical areas that showed a reduction in the contrast of Control $>$ Training, we also conducted a whole-brain analysis comparing the two strategies to examine cortical and sub-cortical structures that were differentially recruited by the two groups at post-assessment. We found greater recruitment of the bilateral primary motor cortices, somatosensory cortices, supplementary motor area, and the posteromedial cortex, including the 


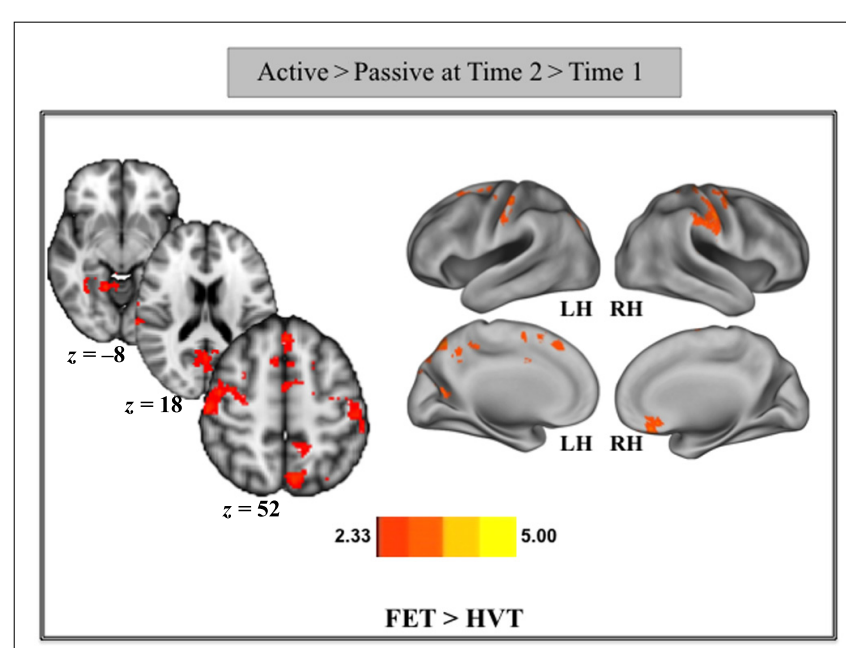

FIGURE 4 | Change in percent signal change from pre- to post-training in the cortical areas found in the contrast of Control $>$ Training for all three groups.

precuneus, and the retrosplenial cortex (see Figure 5 and Table 4) in FET participants post-training, relative to the HVT participants. The contrast of HVT $>$ FET did not result in any significant clusters of activation.

Statistical peaks in these regions were taken to examine associations with behavioral performance across participants, while controlling for the effects of gender. We found a trend for negative associations between game score improvement and increase in activation in the right and the left motor cortices across all participants $(r=-0.22, p=0.08$, and $r=-0.23, p=0.06$ respectively), again suggesting that greater recruitment of the motor cortices with training was associated with poor behavioral improvements on the SF game.

\section{DISCUSSION}

The present study, employing the SF videogame as a context to study multi-tasking and skill acquisition in a complex task, investigated the effects of two types of training strategies in enhancing performance and neural recruitment during videogame play. In line with our hypotheses, we found that videogame training enhanced behavioral performance on a complex task and concurrently reduced the neural demands of SF in areas associated with greater attentional control. In addition, comparing the two training strategies, we found greater training-related improvements associated with HVT relative to FET, along with a reduced need to recruit cortical circuitry subserving executive control and motor performance. Based on these results, HVT is proposed as an effective strategy for accelerating skill acquisition and achieving mastery.

Extensive research supports the utility of repeated practice to enhance behavioral performance (Fabiani et al., 1989; Gopher et al., 1994; Boot et al., 2010). Corroborating these findings, our study reports that repeated exposure to SF leads to higher levels of game mastery in novice videogame players. Across all three groups, participants showed improvement in behavioral performance from pre- to post-training, indicating a beneficial effect of

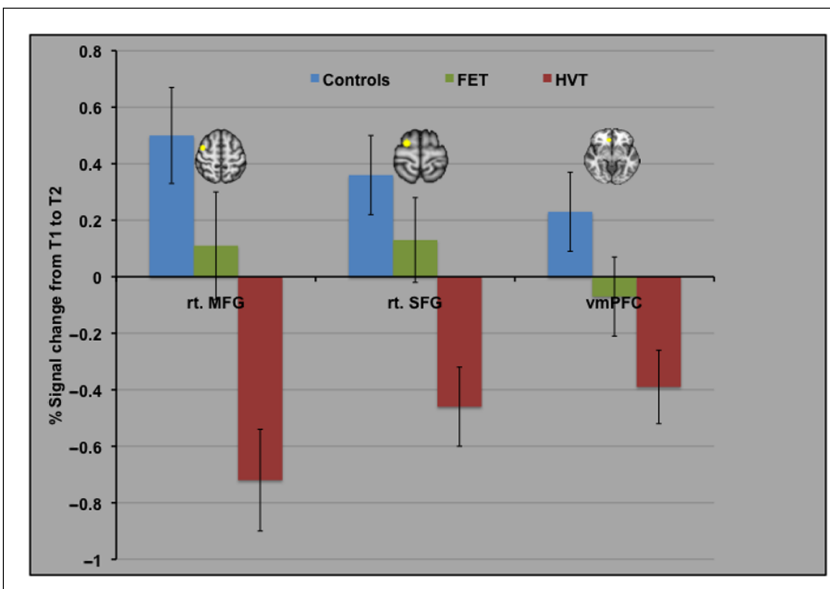

FIGURE 5 | Cortical areas recruited by the FET participants relative to the HVT participants at post-training, when compared to pre-training. All axial slices are presented in radiological orientation.

Table 4 | Statistical peaks of cortical regions recruited during the Active $>$ Passive condition at Time $2>$ Time 1 contrasting the FET group with the HVT group (FET > HVT).

\begin{tabular}{llllll}
\hline Anatomical region & Label & Max & \multicolumn{2}{c}{ MNI coordinates } \\
\cline { 4 - 6 } & & z-stat & & & \\
& & & $\boldsymbol{X}$ & $\boldsymbol{Y}$ & $\boldsymbol{Z}$ \\
\hline Right primary motor cortex & rt. M1 & 2.72 & 42 & -8 & 56 \\
Left primary motor cortex & It. M1 & 2.7 & -44 & -12 & 56 \\
Right postcentral gyrus & Rt. postcentral & 3.56 & 60 & -14 & 40 \\
& gyrus & & & & \\
Left postcentral gyrus & Lt. postcentral & 3.14 & -58 & -18 & 40 \\
& gyrus & & & & \\
Supplementary motor area & SMA & 2.86 & -2 & -4 & 54 \\
Posteromedial cortex & PMC & 2.73 & 2 & -56 & 38
\end{tabular}

basic practice on a complex task (Newell and Rosenbloom, 1981). In addition, we found evidence for superior behavioral performance with a strategy that involved a combination of part-task training, and variable whole-task training, thus adding to the existing literature favoring flexible strategies in acquisition of complex skills relative to constant, repeated practice on the task (Boot et al., 2010; Voss et al., 2011).

To investigate the neural mechanisms associated with videogame training, we also examined the influence of training on functional brain activity. Given that training on the SF game was expected to reduce the attentional demands associated with game-play, we predicted an attenuation of neural activity in areas of the prefrontal and parietal cortices as a result of training. Confirming this hypothesis, we found reduced activation in cortical regions involved in attentional control for the training groups relative to the control group, and also for HVT relative to FET. Specifically, the control group exhibited continued activation in regions of the frontal cortices, including the middle frontal gyrus, and the superior frontal gyrus. These lateral prefrontal regions 
are traditionally known to be involved in processes of top-down control (Miller and Cohen, 2001; Erickson et al., 2009), showing enhanced activation with increasing task demands (Braver et al., 1997; Prakash et al., 2009) and a reduction in activity with relative automaticity of the task (Poldrack et al., 2005). This suggests that the poorer performance of the control group relative to the training groups may, therefore, be related to ineffective control of the joystick during game-play, greater effort in multi-tasking between the different components of the game, and a general enduring need for cortical recruitment in support of task-focused performance. In comparison, the reduced activation of such regions observed in the training groups relative to the control group at post-training represents training-related optimization of neural recruitment during game-play.

An important concept in the acquisition of a complex skill, proposed by Gopher et al. (1989) is the development of higherorder schemas as learners progress through the various stages of skill acquisition and attain mastery of the task. Schemas can be conceptualized as organized series of responses, usually formed after repeated and optimal practice with a task, resulting in efficient performance on the task with minimal resources. The vmPFC is known to be selectively involved in the effortful retrieval of consolidated memory traces that are consistent with pre-existing schemas (van Kesteren et al., 2010), such that greater activation is seen in this region for recall of remote memories, similar to that seen in the hippocampus for recall of recent memories (Frankland and Bontempi, 2005; Takashima et al., 2006). One explanation for the greater activation of the medial prefrontal regions during remote memory recall is the greater effort required to retrieve a degraded and weak schema (Frankland and Bontempi, 2006; Rudy et al., 2006). In our study, we found control participants to show greater activation in the vmPFC than training participants at postintervention, thus, possibly suggesting a failure to form a wellorganized series of responses for the SF game in the control group, resulting in greater neural effort. Training strategies that involve repeated exposure to the game possibly result in the building of higher-order schemas that represent well-organized sequences of responses (Gopher et al., 1989; Kantak et al., 2010). For the control group, due to limited exposure to the SF game, well-organized schemas representing connections between the different elements of the game may not have been built, and thus, we see greater effort being exerted to retrieve a weak memory trace. In contrast, the two training groups did not differ in activation of the medial prefrontal cortices, suggesting that exposure to the game for $30 \mathrm{~h}$ results in the development of higher-order schemas, which can be efficiently retrieved at the time of need.

Thus, whereas game performance on SF led to a persistent taxing of the attentional network, specifically the prefrontal cortices in control participants, individuals in the training groups demonstrated successful performance on a complex task using minimal allocation of attentional resources. In addition, we found continued activation of attentional areas for FET at post-training, which might reflect the inefficient use of two different cognitive control networks in this group (Voss et al., 2011) and their enduring reliance on attentional resources to meet the demands of SF. Our study shows that the attentional costs of multi-tasking, exemplified in lower scores on SF and continual activation of the prefrontal cortices after training (Dove et al., 2000; Gazzaley et al., 2005), are more pronounced for FET than HVT, a finding which predicates the employment of the HVT cognitive training strategy (uniquely involving variable emphasis on different task components combined with basic part-task practice) as a useful approach to improving cognitive functioning. Based on a modest association between game score improvement and decreased activation of the right MFG, we also suggest that such reductions in cortical recruitment, observed in the HVT group, could indeed be related to improved performance on SF. Since decreased recruitment of the cortical regions comprising the attentional network can have implications for behavioral performance, an effective cognitive training tool is one that concurrently hones behavioral skills and optimizes the neural circuitry of attentional control.

Differences in cortical recruitment between the two groups were also seen in the primary motor cortices, the sensory cortices, and the supplementary motor area, with the FET group showing continuing reliance on these areas post-training relative to the HVT group. The involvement of the motor network during the SF game is not surprising given that the control of the ship in the frictionless environment is arguably the most challenging component of this complex task. In fact, greater phasic activity in the right motor cortex during baseline SF play has been found to be beneficial to game performance (Anderson et al., 2011), suggesting that activity in this region is important for learning the game. In fact, both positron-emission tomography (PET) studies (Jenkins et al., 1994; Schlaug et al., 1994) and lesion studies (Pavlides et al., 1993) provide evidence for the involvement of the motor network including the primary motor cortices, the sensorimotor cortices, and the somatosensory cortices in initial acquisition of motor skills, with significant attenuation of activity within the motor network with consolidation of the motor skill as a unitary motor plan (Pascual-Leone et al., 1994). Thus, the continued engagement of the motor network in participants trained under constant practice (FET) suggests the reliance of this strategy on the procedural system, guided by fixed rules and learning. Given that interference with these regions attenuates retention of motor skills following FET (Kantak et al., 2010), suggests the critical involvement of these regions with this practice structure that focuses exclusively on repeated practice of the task, rather than flexible development of strategies and skills that will aid in efficient performance. Indeed, greater recruitment of the primary motor cortices from pre- to post-training was associated with lower gains on the SF task, thus suggesting that individuals demonstrating the greatest improvements in performance as a function of training, also showed a significant decline in their reliance on the motor network.

The addition of neuroimaging techniques provides insight into the influence of videogame training on changes in neural activity during a complex task. Another particular strength of this study is the inclusion of a no-contact control group and a non-VPT group, which previous SF studies have not considered. This is particularly useful because it serves to clarify the confounds present in previous studies between the behavioral and neural characteristics of VPTbased training (HVT) and simple practice effects (FET). For future research it would be important to directly compare the effects of 
the HVT group to that of the variable training, fixed emphasis, and a no-contact control group to truly parse out the effects of the hybrid approach relative to the variable training approach and the fixed emphasis approach. An important limitation of the current study was the collection of neural data after $30 \mathrm{~h}$ of training, as opposed to assessing changes in neural functioning after a shorter period of training. Since differences in behavioral performance between HVT and FET were predominant after $10 \mathrm{~h}$ of training (reported in Lee et al., 2012), we suspect that $30 \mathrm{~h}$ of videogame training may have not entirely captured neural differences between the two training groups when performance differences were at their maximum. Although a comparison of the training groups in this study indicates a significant advantage for participants in the HVT group, this advantage could potentially be more evident if measured earlier in training. Therefore, future studies examining changes in neural recruitment earlier in training would be critical to understanding the dose-response relationship between training and neural recruitment.

Previous studies of attentional and executive control have established that cortical recruitment of the regions comprising the attentional network is responsive to task demands (Banich et al., 2000; Dove et al., 2000). However, the role of this additional neural activation has been disputed, with some studies suggesting that activation may serve a compensatory function (Davis et al., 2008), while others argue that excessive attentional network activation is related to diminished performance on a cognitive task (Gazzaley et al., 2005; Prakash et al., 2009). The aging literature, for example, has associated extensive cortical recruitment in older adults with poorer performance on a cognitive task (Prakash et al., 2009; see also Schneider-Garces et al., 2010). Thus, the implementation of a randomized controlled trial similar to the one used in the present study could shed light on the neural correlates

\section{REFERENCES}

Ackerman, P. L. (1988). Determinants of individual differences during skill acquisition: cognitive abilities and information processing. J. Exp. Psychol. Gen. 117, 288-318.

Anderson, J. R., Bothell, D., Fincham, J. M., Anderson, A. R., Poole, B., and Qin, Y. (2011). Brain regions engaged by part- and whole-task performance in a video game: a model-based test of the decomposition hypothesis. J. Cogn. Neurosci. 23, 3983-3997.

Banich, M. T., Milham, M. P., Atchley, R. A., Cohen, N. J., Webb, A., Wszalek, T., Kramer, A. F., Liang, Z., Barad, V., Gullett, D., Shah, C., and Brown, C. (2000). Prefrontal regions play a predominant role in imposing an attentional 'set': evidence from fMRI. Brain Res. Cogn. Brain Res. 10, 1-9.

Basak, C., Boot, W. R., Voss, M., and Kramer, A. F. (2008). Can training in a real-time strategy videogame attenuate cognitive decline in older adults? Psychol. Aging 23, 765-777.

Bherer, L., Kramer, A. F., Peterson, M. S., Colcombe, S., Erickson, K., and Becic,

associated with improved executive function in older adults and represents a potentially interesting and valuable study for future investigations.

In summary, the present study provides evidence for the ability of videogame training to enhance performance on a complex task and correspondingly decrease cortical recruitment of attentional resources. Based on behavioral and neuroimaging evidence, we conclude that HVT, relative to FET, may facilitate greater mastery of a complex task and neural efficiency in response to task difficulty. In general, videogame training signifies a novel and promising avenue to improving cognition and maximizing efficiency in neural recruitment, thereby making it a plausible tool for use with clinical populations in the future.

\section{ACKNOWLEDGMENTS}

This study was supported by grant from the Office of Naval Research (Grant Number N000140710903). We would like to thank Matt van Patter, Jason Lewis, Zuha Warraich, Dawn Epstein, Sarah Dalton, Matt Windsor, Maulik Sheth, Yasuo Kubota, Shanna Desouza, Holly Tracey, and Nancy Dodge for their help in data collection.

\section{AUTHOR CONTRIBUTIONS}

Hyunkyu Lee, Michelle W. Voss, Walter R. Boot, Chandramallika Basak, Monica Fabiani, Gabriele Gratton, Arthur F. Kramer, and Ruchika S. Prakash designed the behavioral and fMRI components of the study. Hyunkyu Lee, Michelle W. Voss, Walter R. Boot, and Ruchika S. Prakash collected the imaging data. Ruchika S. Prakash, Angeline A. De Leon, Lyla Mourany, Hyunkyu Lee, and Michelle W. Voss analyzed the behavioral and imaging data. Ruchika S. Prakash and Angeline A. De Leon wrote the paper and all co-authors edited the manuscript.

memory performance in multiple sclerosis: a randomized clinical trial. Mult. Scler. 11, 58-68.

Craik, F. I., Govoni, R., NavehBenjamin, M., and Anderson, N. D. (1996). The effects of divided attention on encoding and retrieval processes in human memory. J. Exp. Psychol. Gen. 125, 159-180.

Davis, S. W., Dennis, N. A., Daselaar, S. M., Fleck, M. S., and Cabeza, R. (2008). Que PASA? The posterioranterior shift in aging. Cereb. Cortex 18, 1201-1209.

Donchin, E., Fabiani, M., and Sanders, A. (1989).The Learning Strategies Program: An Examination of the Strategies in Skill Acquisition. Amsterdam: Elsevier.

Dove, A., Pollmann, S., Schubert, T., Wiggins, C. J., and von Cramon, D. Y. (2000). Prefrontal cortex activation in task switching: an event-related fMRI study. Brain Res. Cogn. Brain Res. 9, 103-109.

Erickson, K. I., Colcombe, S. J., Wadhwa, R., Bherer, L., Peterson, M. S., Scalf, P. E., Kim, J. S., Alvardo, M., and Kramer, A. F. (2007).
Training-induced functional activation changes in dual-task processing: an fMRI study Cereb. Cortex 17, 192-204.

Erickson, K. I., Prakash, R. S., Kim, J. S., Sutton, B. P., Colcombe, S. J., and Kramer, A. F. (2009). Topdown attentional control in spatially coincident stimuli enhances activity in both task-relevant and taskirrelevant regions of cortex. Behav. Brain Res. 197, 186-197.

Fabiani, M., Buckley, J., Gratton, G., Coles, M. G. H., Donchin, E., and Logie, R. (1989).The training of complex task performance. Acta Psychol. 71, 259-299.

Feng, J., Spence, I., and Pratt, J. (2007). Playing an action video game reduces gender differences in spatial cognition. Psychol. Sci. 18, 850-855.

Fitts, P., and Posner, M. I. (1967). Human Performance. Belmont, CA: Brooks/Cole.

Frankland, P. W., and Bontempi, B. (2005). The organization of recent and remote memories. Nat. Rev. Neurosci. 6, 119-130. 
Frankland, P. W., and Bontempi, B. (2006). Fast track to the medial prefrontal cortex. Proc. Natl. Acad. Sci. U.S.A. 103, 509-510.

Frederiksen, J. R., and White, B. Y. (1989). An approach to training based upon principled task decomposition. Acta Psychol. 71, 89-146.

Gazzaley, A., Cooney, J. W., McEvoy, K., Knight, R. T., and D'Espsito, M. (2005). Top-down enhancement and suppression of the magnitude and speed of neural activity. J. Cogn. Neurosci. 17, 507-517.

Gopher, D., Weil, M., and Bareket, T. (1994). Transfer of skill from a computer game trainer to flight. Hum. Factors 36, 387-405.

Gopher, D., Weil, M., and Siegel, D. (1989). Practice under changing priorities: an approach to the training of complex skills. Acta Psychol. 71, 147-177.

Jenkins, I. H., Brooks, D. J., Nixon, P. D., Frackowiak, R. S. J., and Passingham, R. E. (1994). Motor sequence learning: a study with positron emission tomography. J. Neurosci. 14, 3775-3790.

Kantak, S. S., Sullivan, K. J., Fisher, B. E., Knowlton, B. J., and Winstein, C. J. (2010). Neural substrates of motor memory consolidation depend on practice structure. Nat. Neurosci. 13, 923-925.

Kramer, A. F., Larish, J. F., and Strayer, D. L. (1995). Training for attentional control in dual task settings: a comparison of young and old adults. J. Exp. Psychol. Appl. 1, 50-76.

Kramer, A. F., Larish, J. F., Weber, T., and Bardell, L. (1999). "Training for executive control: task coordination strategies and aging," in Attention and Performance XVII, eds D. Gopher and A. Koriat (Cambridge, MA: MIT Press), 617-652.

Lee, H., Boot, W. R., Basak, C., Voss, M. W., Prakash, R. S., Erickson, K. I., Neider, M., Simons, D. J., Fabiani, M., Gratton, G., Low, K. A., and Kramer, A. F. (2012). Effective training of complex tasks: how strategy and individual differences contribute to performance, improvement, and transfer of training. Acta Psychol. 139, 146-158.

Maclin, E. L., Mathewson, K. E., Low, K. A., Boot, W. R., Kramer, A. F., Fabiani, M., and Gratton, G. (2011). Learning to multitask: effects of video game practice on electrophysiological indices of attention and resource allocation. Psychophysiology 48, 1173-1183.

Mané, A., and Donchin, E. (1989). The Space Fortress game. Acta Psychol. 71, 17-22.

Miller, E. K., and Cohen, J. D. (2001). An integrative theory of prefrontal cortex function. Annu. Rev. Neurosci. 24, 167-202.

Myers, C. E., Shohamy, D., Gluck, M. A., Grossman, S., Kluger, A., Ferris, S., Golomb, J., Schnirman, G., and Schwartz, R. (2003). Dissociating hippocampal versus basal ganglia contributions to learning and transfer. J. Cogn. Neurosci. 15, 185-193.

Newell, A., and Rosenbloom, P. S. (1981). "Mechanisms of skill acquisition and the law of practice," in Cognitive Skills and Their Acquisition, ed. J. R. Anderson (Hillsdale, NJ: Lawrence Erlbaum Associates, Inc.), 1-55.

Olesen, P., Westerberg, H., and Klingberg, T. (2004). Increased prefrontal and parietal activity after training of working memory. Nat. Neurosci. 7, 75-79.

Pascual-Leone, A., Grafman, J., and Hallett, M. (1994). Modulation of cortical motor output maps during development of implicit and explicit knowledge. Science 263, 1287-1289.

Pavlides, C., Miyashita, E., and Asanuma, H. (1993). Projection from the sensory to the motor cortex is important in learning motor skills in monkeys. J. Neurophysiol. 70, 733-741.

Piaget, J. (1954). The Construction of Reality in the Child. New York: Basic Books.

Poldrack, R. A., Sabb, F. W., Foerde, K., Tom, S. M., Asarnow, R. F., Bookheimer, S. Y., and Knowlton, B. J. (2005). The neural correlates of motor skill automaticity. J. Neurosci. 25, 5356-5364.

Prakash, R. S., Erickson, K. I., Colcombe, S. J., Kim, J. S., Voss, M.
W., and Kramer, A. F. (2009). Agerelated differences in the involvement of the prefrontal cortex in attentional control. Brain Cogn. 71, 328-335.

Rabbitt, P., Banerji, N., and Szemanski, A. (1989). Space Fortress as an IQ test? Predictions of learning and practiced performance in a complex interactive video-game. Acta Psychol. 71, 243-257.

Rudy, J. W., Biedenkapp, J. C., and O'Reilly, R. (2006). Prefrontal cortex and the organization of recent and remote memories: an alternative view. Learn. Mem. 12, 445-446.

Schlaug, G., Knorr, U., and Seitz, R. J. (1994). Inter-subject variability of cerebral activations in acquiring a motor skill: a study with positron emission tomography. Exp. Brain Res. 98, 523-534.

Schneider, W., and Shiffrin, R. M. (1977). Controlled and automatic human information processing: II. Perceptual learning, automatic attending, and a general theory. Psychol. Rev. 84, 127-190.

Schneider-Garces, N. J., Gordon, B. A., Brumback-Peltz, C. R., Shin, E. Lee, Y., Sutton, B. P., Maclin, E. L., Gratton, G., and Fabiani, M. (2010). Span, CRUNCH and beyond: working memory capacity and the aging brain. J. Cogn. Neurosci. 22, 655-669.

Slagter, H. A., Davidson, R. J., and Lutz, A. (2011). Mental training as a tool in the neuroscientific study of brain and cognitive plasticity. Front. Hum. Neurosci. 5:17. doi: 10.3389/fnhum.2011.00017

Smith, M. E., McEvoy, L. K., and Gevins, A. (1999). Neurophysiological indices of strategy development and skill acquisition. Cogn. Brain Res. 7, 389-404.

Stern, Y., Blumen, H. M., Rich, L. W., Richards, A., Herzberg, G., and Gopher, D. (2011). Space Fortress game training and executive control in older adults: a pilot intervention. Neuropsychol. Dev. Cogn. B Aging Neuropsychol. Cogn. 18, 653-677.

Takashima, A., Petersson, K. M., Rutters, F., Tendolkar, I., Jensen, O. Zwarts, M. J., McNaughton, B. L., and Fernandez, G. (2006). Declarative memory consolidation in humans: a prospective functional magnetic resonance imaging study. Proc. Natl. Acad. Sci. U.S.A. 103, 756-761.

Terlecki, M. S., and Newcombe, N. S. (2005). How important is the digital divide? The relation of computer and videogame usage to gender differences in mental rotation ability. Sex Roles 53, 433-441.

van Kesteren, M. T. R., Rijpkema, M., Ruiter, D. J., and Fernandez, G. (2010). Retrieval of associative information congruent with prior knowledge is related to increased medial prefrontal activity and connectivity. J. Neurosci. 30, 15888-15894.

Voss, M. W., Prakash, R. S., Erickson, K. I., Boot, W. R., Basak, C., Neider, M. B., Simons, D. J., Fabiani, M., Gratton, G., and Kramer, A. F. (2011). Effects of training strategies implemented in a complex videogame on functional connectivity of attentional networks. Neuroimage 59, 138-148.

Conflict of Interest Statement: The authors declare that the research was conducted in the absence of any commercial or financial relationships that could be construed as a potential conflict of interest.

Received: 02 December 2011; paper pending published: 05 January 2012; accepted: 14 April 2012; published online: 15 May 2012.

Citation: Prakash RS, De Leon AA, Mourany L, Lee H, Voss MW, Boot WR, Basak C, Fabiani M, Gratton $G$ and Kramer AF (2012) Examining neural correlates of skill acquisition in a complex videogame training program. Front. Hum. Neurosci. 6:115. doi: 10.3389/ fnhum.2012.00115

Copyright (c) 2012 Prakash, De Leon, Mourany, Lee, Voss, Boot, Basak, Fabiani, Gratton and Kramer. This is an open-access article distributed under the terms of the Creative Commons Attribution Non Commercial License, which permits non-commercial use, distribution, and reproduction in other forums, provided the original authors and source are credited. 